Revista Destaques Acadêmicos, Lajeado, v. 12, n. 4, 2020. ISSN 2176-3070

DOI: http://dx.doi.org/10.22410/issn.2176-3070.v12i4a2020.2389

http://www.univates.br/revistas

\title{
IMPLEMENTAÇÃO DE SISTEMA DE VIDEOMONITORAMENTO DE BAIXO CUSTO UTILIZANDO ZONEMINDER E CÂMERAS IP
}

\author{
Robinson Stürmer ${ }^{1}$, Edson Moacir Ahlert ${ }^{2}$
}

Resumo: Os atuais índices de violência têm aumentado a procura por soluções de segurança, sendo que sistemas de CFTV (Circuito fechado de televisão) são uma das soluções utilizadas. Este trabalho tem por finalidade a implementação de um protótipo de baixo custo com armazenamento remoto das imagens em nuvem, gerenciamento centralizado utilizando conceitos que englobam as áreas do curso de Redes de Computadores como a utilização de comunicação entre os dispositivos com os protocolos TCP/IP (Protocolo de controle de transmissão e Protocolo Internet), roteamento estático, VPN (Rede privada virtual), processamento distribuído de informações, alta disponibilidade, criação de rotinas automatizadas em Linux Bash, expressões regulares e serviço de monitoramento de rede, detectando anomalias e performance dos dispositivos do sistema. Para que o objetivo fosse atingido foram necessárias modificações do cenário inicial, incluindo dispositivo remoto para que as imagens fossem processadas e enviadas somente quando necessário. A solução mostrou-se viável e suficiente para diversas aplicações, pois além do baixo custo para aquisição dos equipamentos, seu custo de manutenção também é baixo.

Palavras-chaves: CFTV, Zoneminder, Raspberripy.

\section{INTRODUÇÃO}

$\mathrm{O}$ avanço tecnológico tem proporcionado uma série de convergências das tecnologias de segurança eletrônica do meio analógico para o digital e IP. Essa convergência está permitindo a implantação de sistemas de baixo custo, bem como a criação de centros de monitoramento e segurança patrimonial, com sistemas de gerenciamento centralizado e remoto, possibilitando o recebimento de informações instantaneamente utilizando a internet como infraestrutura de

1 Tecnólogo em Redes de Computadores. E-mail: robin_stu@universo.univates.br

2 Mestre em Ambiente e Desenvolvimento. Professor da Univates. E-mail: edsonahlert@univates.br 
comunicação, o que proporciona um potencial ainda maior para esse tipo de tecnologia.

A crescente onda de violência na atualidade, em todo território nacional, fez com que a procura por soluções de CFTV aumentasse. Aliado a este fator, a grande expansão da internet, que facilita o acesso à informação, permitindo que haja a disseminação de conhecimento sobre soluções tecnológicas do ramo de vigilância eletrônica, vêm contribuindo para este aumento de demanda e disponibilidade dessas tecnologias no mercado. A implantação de videomonitoramento em residências, empresas e até em vias públicas tem contribuído para o aumento de segurança, muitas vezes diminuindo a incidência de crimes ou ainda proporcionando a elucidação de diversas ocorrências (CORREA NETO, 2009).

Apesar da popularização desse tipo de tecnologia, a convergência da comunicação entre os dispositivos para o meio digital ainda sofre alguns entraves. Devido a falta de profissionais de CFTV no mercado com conhecimento de redes de computadores, a consequência, muitas vezes, é a tentativa de utilização de equipamentos analógicos, limitando ou até mesmo inviabilizando a elaboração ou implantação de projetos de vigilância confiáveis. Desta forma, o conhecimento de redes, como a definição de alguns conceitos sobre IP, máscara de rede, roteamento de rede, encaminhamento de portas, NAT, firewall, QoS, VLAN entre outros, é cada vez mais importante.

Outro fator a ser considerado na implementação do serviço de videomonitoramento é a terceirização deste, por meio de empresas especializadas. Apesar de apresentar-se como uma solução bastante fácil e gerar uma sensação de segurança, sua implantação geralmente não contempla algumas características, tais como o armazenamento remoto de imagens e monitoramento do status do sistema. A ausência destas funcionalidades pode torná-lo inefetivo, frustrando o cliente por não ter atingido o objetivo para o qual foi contratado.

Um exemplo típico dessa situação é a expectativa gerada na contratação de um serviço de videomonitoramento, onde geralmente é subentendido que as imagens estarão sendo acompanhadas vinte e quatro horas por dia. Ao ser registrado uma ocorrência, na qual é subtraído do local monitorado o equipamento centralizador de imagens, descobre-se que o serviço pago foi em vão.

A maioria das ferramentas disponíveis no mercado até possuem alguns recursos de armazenamento remoto, como envio de imagens por meio de protocolos como SMTP (Protocolo de transferência de correio simples) e FTP (Protocolo de Transferência de Arquivos), mas muitas vezes são funções bastante limitadas, devida a quantidade de registros enviados por evento. A grande maioria dos provedores de serviço de e-mail limitam a quantidade de mensagens que podem ser enviadas por um certo período, por questões de segurança, o que pode gerar o bloqueio da conta de e-mail em dias de 
alta incidência de detecção de movimento, inviabilizando este método de armazenamento.

Essa grande incidência de registros pode ocorrer devido a geração de falsos-positivos, como em um dia em que o sol tem intensidade de iluminação variada em função da movimentação de nuvens. Outra questão, é possível que na área monitorada excepcionalmente ocorra um aumento de movimentação, como a exemplo da colocação de um varal de roupa portátil em dias com existência de vento. Por sua vez, a utilização do protocolo FTP para o armazenamento das imagens remotamente, além da questão do espaço disponível para o armazenamento, há a necessidade de que o serviço seja publicado em um endereço IP público, podendo gerar custos e onerando a implantação do sistema.

Com a utilização de software livre para solução do assunto proposto, pode-se obter uma maior flexibilidade, possibilitando um aumento da disponibilização de funcionalidades habilitadas no sistema, agregadas através da instalação de outros aplicativos.

Para a questão do armazenamento remoto, aproveitando-se da expansão de serviços de armazenamento em nuvem como o Google Drive, Dropbox e Microsoft Onedrive, pode-se utilizar softwares como o Rclone para sincronização de arquivos. Este software também permite a integração com diversas outras plataformas de armazenamento em nuvem, baseado no software de sincronização Rsync, ele permite sincronizar dados em ambos os sentidos, bem como a remoção automática dos dados enviados após um período especificado (RCLONE, 2018).

Atualmente, a infraestrutura de rede da telefonia móvel permite a utilização de internet banda larga de alta velocidade, chegando facilmente em taxas de transmissão na casa dos $20 \mathrm{Mbps}$ para download e $10 \mathrm{Mbps}$ de upload. Estas taxas de transmissão permitem tranquilamente o envio de streaming de vídeo em HD (High definition, resolução de 1280 x 720p). Outro fator positivo é a aquisição desse tipo de plano de dados, com pacotes na modalidade prépago chegando a 8 GBytes de consumo mensal, com um custo muito baixo, chegando a custar 50\% do valor de uma internet fixa (CLARO, 2018).

Apesar do ótimo custo benefício dos planos atuais de internet móvel, o consumo ofertado ainda não é o suficiente para estabelecer uma conexão entre uma câmera remota a um centralizador de imagens, como um gravador NVR (Gravador de vídeo digital) ou servidor, com fluxo constante das imagens da câmera ao centralizador de imagens. Uma das formas encontradas para contornar essa limitação é a utilização de processamento de imagens distribuído, instalando no local remoto, a mesma aplicação de videomonitoramento, porém compartilhando a base de dados e repositório de imagens. Nesta estratégia de utilização de processamento distribuído das imagens, ocorre um fluxo de dados quando há detecção de movimento, gerando otimização do consumo de internet. 
O objetivo deste trabalho é apresentar e analisar os resultados da implementação de uma solução de CFTV de baixo custo, que permita um gerenciamento centralizado e remoto, utilizando processamento distribuído, serviços de rede como armazenamento remoto das informações, aplicando camada de VPN (Rede privada virtual) para viabilizar uma comunicação remota com um nível de segurança, utilizando software livre e equipamentos de baixa capacidade de processamento e baixo consumo de energia, para aplicação do sistema em locais remotos com acesso a redes de dados móveis e a possibilidade de alimentação por energia elétrica gerada de forma alternativa, como eólica ou solar.

\section{REFERENCIAL TEÓRICO}

Nesta seção é apresentado o referencial teórico, onde são demonstradas e explicadas as tecnologias relacionadas à CFTV e termos relacionados, utilizadas na execução do presente trabalho.

\subsection{ZoneMinder}

O ZoneMinder é um software de código aberto e gratuito. Ele é formado por um conjunto integrado de aplicativos, fornecendo uma solução completa de CFTV. Este software permite captura, análise e gravação de imagens, de forma contínua, por detecção de movimento ou por acionamento de gatilhos (ZONEMINDER, 2018).

Devido às múltiplas formas de acesso ao Zoneminder, possibilita que ele seja utilizado a partir de qualquer dispositivo conectado à internet que possua um navegador web, bem como por aplicativos para Android e IOS. Na versão atual, 1.30.4 é possível acessar todas opções de configurações disponíveis na interface web através de um celular (ZONEMINDER, 2018).

Outro benefício do Zoneminder é a possibilidade da adição de quase todos os tipos de câmeras, sejam elas USB ou analógicas, tendo com restrição dispositivos que não possuam driver para o sistema operacional Linux em que o sistema foi instalado. Também é possível a utilização de câmeras IP compatíveis com o padrão Onvif, protocolos RTSP, HTTPS e função PTZ (ZONEMINDER, 2018).

Uma das características principais do Zoneminder é a sua versatilidade, possibilitando a implantação de um sistema pequeno de CFTV, como uma casa ou escritório, bem como a criação de sistemas com inúmeras câmeras, vários servidores de análise, armazenamento de dados e imagens, permitindo o processamento distribuído das informações coletadas pelo sistema implantado, conforme demonstrado na Figura 1. 
Figura 1: Topologia de rede Zoneminder Multi-Server

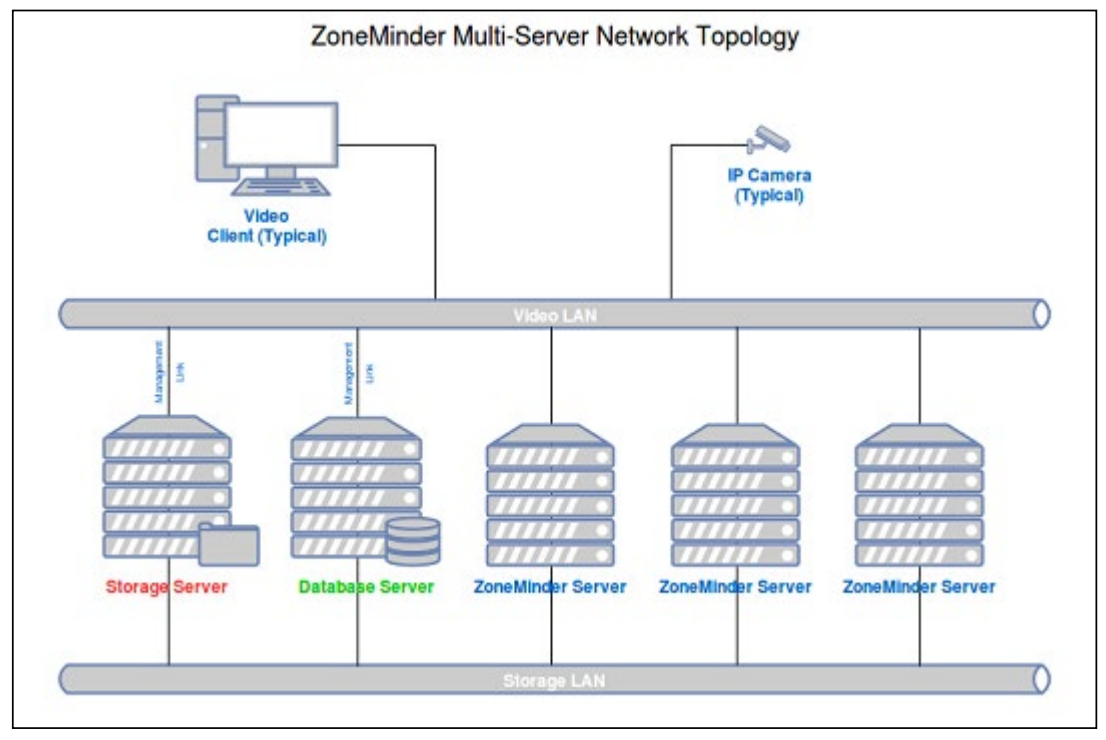

Fonte: Zoneminder (2018).

Igualmente é possível realizar uma gama de personalizações, o que aumenta ainda mais suas capacidades, como a adição de mais aplicativos de replicação de dados a outro servidor, replicação de dados com plataforma de armazenamento em nuvem, acesso remoto, aplicativos de monitoramento de serviços, envio de e-mails e SMS de notificações (ZONEMINDER, 2018).

A funcionalidade Multi-server do Zoneminder permite que um servidor instalado em um prédio remoto monitore diversas câmeras e envie imagens quando acionado por gatilhos ou detecção de movimento, otimizando assim o uso do link de internet, possibilitando a utilização de internet móvel.

\subsection{Nagios Core}

O Nagios Core é um sistema código aberto de monitoramento de dispositivos e serviços de rede, permitindo analisar, identificar e resolver problemas antes que causem impactos críticos ao negócio. Além de possibilitar fornecimento de monitoramento completo da infraestrutura de TI, incluindo desde sistemas operacionais, aplicativos, serviços até toda infraestrutura de rede.

Uma importante característica deste aplicativo, permite a organização planejar e melhorar sua infraestrutura, pois com os relatórios é possível verificar se as SLAs estão sendo atendidos em tempo hábil, também proporcionando um histórico com registros de quedas de serviços ou dispositivos até seu restabelecimento. 
O Nagios possui envio de notificação multiusuário, possibilitando enviar alertas para equipe de TI por e-mail ou SMS. Os eventos podem ser personalizados e enviados a equipe técnica responsável por determinada área, permitindo inclusive a integração de outros softwares do mesmo desenvolvedor e de terceiros (NAGIOS, 2018).

\subsection{Minicomputadores embarcados}

Os minicomputadores embarcados inicialmente foram criados para fomentar conhecimento de ciência da computação pela instituição Raspberry Pi Foundation, no Reino Unido. Com um tamanho aproximado de um cartão de crédito, aliado a um ótimo desempenho e preço baixo, o dispositivo teve bastante sucesso. Com isso passaram a ser utilizado em diversas aplicações, de entretenimento a até aplicações de automação residencial (RASPBERRYPI, 2018).

O sucesso desta plataforma fez com que surgissem no mercado outros dispositivos semelhantes de diferentes fabricantes (GOGONI, 2016), aumentando ainda mais o seu emprego em diversas aplicações (SIMÕES, 2016). Entre os principais dispositivos disponíveis no mercado podemos citar o Raspberry Pi, Orange Pi, Banana Pi, Cubieboard, Odroid. Há diversos modelos, com faixas de preços variando entre U\$ 10,00 a U\$ 40,00.

Podem ser utilizados para diversas aplicações, desde um computador para um escritório, um mini servidor para determinada aplicação, até um videogame. Como o sistema operacional destes dispositivos geralmente é armazenado em um cartão SD, possibilita trocar a sua função rapidamente, transformando-o de um computador para um videogame, por exemplo. A gama de sistemas operacionais varia conforme o modelo do minicomputador utilizado, neste trabalho será utilizado um Raspberry PI 3 B e um Orange PI One (FINATI, 2016).

\subsection{Rclone}

O Rclone tem a função sincronizar arquivos e diretórios com diversos serviços de armazenamento em nuvem, FTP, SFTP e armazenamento local. O Rclone é baseado no software Rsync, ele foi projetado para funcionar com serviços em nuvem sem a necessidade de configurar o rsync nas máquinas remotas. Alguns dos serviços de armazenamento em nuvem que ele trabalha são o Google Drive, o Amazon S3, o Dropbox, o Google Cloud Storage, o Amazon Drive, o Microsoft One Drive, o Hubic e o Backblaze B2, totalizando 35 opções de sincronização (RCLONE, 2018).

Neste trabalho, este software será utilizado para fins de armazenamento das imagens remotas capturadas pelo Zoneminder, tanto no centralizador de imagens, quanto no Zoneminder remoto. Neste último, o Rclone será utilizado somente para sincronizar as imagens quando perder acesso a base de dados 
do Zoneminder central, armazenando as imagens no Google Drive. Já no Zoneminder, rodando no centralizador das imagens, será executado de forma contínua, a fim de garantir cópia das imagens armazenadas fora do dispositivo. O tempo de armazenamento pode ser ajustado conforme a necessidade do usuário, ou capacidade, sendo possível a remoção automática das imagens após período de tempo especificado em comando do Rclone.

\subsection{VPN}

Uma Rede Privada Virtual (VPN) é uma rede que tem por objetivo interligar redes corporativas de forma segura através da internet, ou ainda fornecer acesso seguro a usuários remotos. As VPNs são conexões ponto-aponto, criando túneis criptografados entre os dispositivos (KUROSE; ROSS, 2015).

Além da segurança proporcionada pela VPN, outra vantagem é que este serviço substitui a necessidade de contratação de links dedicados para interligação de redes distantes.

Neste trabalho optou-se pela utilização de VPN com tunelamento de Nível 2 PPTP, da Microsoft, pela facilidade de implementação nos equipamentos roteadores da marca Mikrotik. Também foi considerado que somente a conexão de internet na base de armazenamento possui IP público. Outro aspecto vantajoso é que a conexão de internet do local remoto monitorado não necessita de IP público, tornando o custo da contratação deste menor.

\section{MATERIAIS E MÉTODOS}

Neste capítulo são relacionados os materiais, como equipamentos, dispositivos, softwares e técnicas para implementação de um protótipo de CFTV com processamento distribuído, otimização do uso de conexão de dados e gerenciamento centralizado.

Quanto aos objetivos esta pesquisa é exploratória, por proporcionar maior familiaridade com o problema e quanto aos procedimentos técnicos é bibliográfica, pois está alicerçada em material já elaborado, constituído principalmente de livros e artigos científicos (GIL, 2008).

Com a prova de conceito é possível demonstrar, dentro do ambiente proposto, as funcionalidades do estudo em questão dentro da metodologia abordada. Neste estudo, a proposta é utilizar o CFTV digital com software livre e integrá-lo às funcionalidades existentes nos serviços de telefonia celular atuais, objetivando maior flexibilidade e agilidade. Nesta prova de conceito serão materializadas as seguintes etapas do projeto:

- CFTV digital em software livre;

- Disponibilização das imagens na WEB independente do tipo de IP, público ou privado; 
- Visualização das imagens capturadas no aparelho de telefonia móvel celular;

- Envio de e-mails para remetentes cadastrados sinalizando detecção de movimento ocorridas;

- Utilização de uma interface cliente, permitindo a visualização das imagens das câmeras em celulares específicos e de forma contínua.

Para implementação do projeto foram utilizadas as seguintes soluções em equipamentos e aplicativos: Raspberry Pi3; Orange Pi One; Câmera IP Intelbras VIP 1120 D; Mikrotik; Lubuntu Desktop 16.04 no Raspberry PI 3; Armbian Desktop v4.14 no Orange Pi One; Zoneminder; Rclone; Nagios Core e Rotinas Bash. Na Figura 2 estão demonstradas as especificações das soluções aplicadas no projeto de videomonitoramento.

Figura 2: Especificações técnicas dos equipamentos e aplicativos

\begin{tabular}{|c|c|}
\hline $\begin{array}{l}\text { RASPBERRY PI 3+ } \\
\text { - Processador arquitetura arm Quad Core } 1.2 \mathrm{Ghz} \\
\text { - } 1 \mathrm{~GB} \text { de memória Ram } \\
\text { - Wireless LAN } \\
\text { - Fonte de alimentação } 5 \mathrm{v}, 2.5 \mathrm{~A} \\
\text { - Ethernet } 100 \mathrm{Mb} / \mathrm{s} \\
\text { - Saída de Vídeo FullHD } \\
\text { - Soquete para cartão de memória Micro SD } \\
\text { - Utilizado catão memória de } 32 \mathrm{~GB}\end{array}$ & $\begin{array}{l}\text { ORANGEPI ONE } \\
\text { - Processador arquitetura arm Quad Core } 1.2 \mathrm{Ghz} \\
\text { - } 512 \mathrm{MB} \text { de memória Ram } \\
\text { - Fonte de alimentação } 5 \mathrm{v}, 2 \mathrm{~A} \\
\text { - Ethernet } 100 \mathrm{Mb} / \mathrm{s} \\
\text { - Saida de Video resolução } 4 \mathrm{~K} \\
\text { - Soquete para cartão de memória Micro SD } \\
\text { - Utilizado cartão memória de16GB }\end{array}$ \\
\hline $\begin{array}{l}\text { CÂMERA IP INTELBRAS VIP } 1120 \text { D } \\
\text { - Resolução } 720 p(1280 \times 720) \\
\text { - Ângulo de visão H: } 80,36^{\circ} \text { V: } 45,66^{\circ} \\
\text { - Protocolos TCP/IP, IPv4, DHCP, DNS, RTSP, } \\
\text { Filtro IP, DDNS, SMTP, TLS, FTP, NTP, Onvif } \\
\text { - Ethernet } 100 \mathrm{Mb} / \mathrm{s} \\
\text { - } \text { Acesso por Cloud }\end{array}$ & $\begin{array}{l}\text { MIKROTIK HAP } 951 \\
\text { - Processador arquitetura MIPSBE single Core } 650 \\
\text { - } 64 \mathrm{MB} \text { de memória Ram } \\
\text { - Fonte de alimentação } 24 \mathrm{v}, 0.8 \mathrm{~A} \\
\text { - } 4 \text { portas Ethernet } 100 \mathrm{Mb} / \mathrm{s} \\
\text { - } \text { Wireless Lan } 802.11 \mathrm{~b} / \mathrm{g} \\
\text { - Armazenamento interno de } 16 \mathrm{MB} \\
\text { - USB Tipo A, com função USB Power Reset }\end{array}$ \\
\hline $\begin{array}{l}\text { LUBUNTU DESKTOP } \\
\text { - Baseado no Ubuntu } \\
\text { - Código aberto } \\
\text { - Projetado para baixo consumo de energia } \\
\text { - Otimizado para hardwares de baixa capacidade } \\
\text { - Possui um grade de apps leves, como office, } \\
\text { - Área de trabalho baseada em LXDE } \\
\text { - Permite acesso remoto, por SSH e VNC }\end{array}$ & $\begin{array}{l}\text { ARMBIAN DESKTOP } \\
\text { - Linux baseado em Debian com um poderoso } \\
\text { configurador e instalador de software. } \\
\text { - Código aberto } \\
\text { - Projetado para baixo consumo de energia } \\
\text { - Otimizado para hardwares de baixa capacidade } \\
\text { - Area de trabalho baseada em XFCE } \\
\text { - Suporte a vários chips e placas de } \\
\text { - Permite acesso remoto, por SSH e VNC }\end{array}$ \\
\hline
\end{tabular}

Fonte: Dos autores (2018). 
Na primeira tentativa de implantação do sistema de videomonitoramento, utilizando o software Zoneminder, o cenário estava disposto conforme a Figura 3. Apesar do êxito no funcionamento, a transmissão contínua das imagens do local remoto monitorado até o Zoneminder resultaram no consumo do pacote de dados disponível de 1 GByte/semana em apenas algumas poucas horas, sendo que a solução desse problema se tornou um dos tópicos necessário a ser contornado neste trabalho. Os testes dos cenários iniciaram em outubro de 2017, a partir desta data foram sendo implementadas as demais funcionalidades até a concepção final atual.

Figura 3: Cenário 1, topologia da primeira tentativa de implantação do Zoneminder

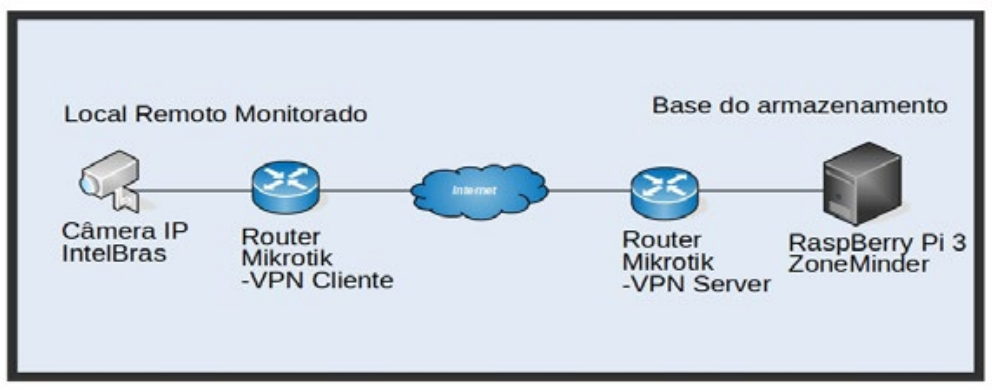

Fonte: Dos autores (2018).

A Figura 4 demonstra a ligação lógica do sistema na primeira tentativa de implementação. Neste formato a câmera de segurança instalada no local remoto monitorado está fornecendo o streaming de imagens para o RaspBerry PI, que executa o software Zoneminder, instalado na base de armazenamento. Os dispositivos estão configurados com endereço IP de redes distintas, na câmera de segurança está configurado o IP 192.168.85.5/24 e no Zoneminder está configurado o IP 192.168.88.10/24.

Figura 4: Cenário 2, rede após utilização de um Zoneminder Server na estrutura remota

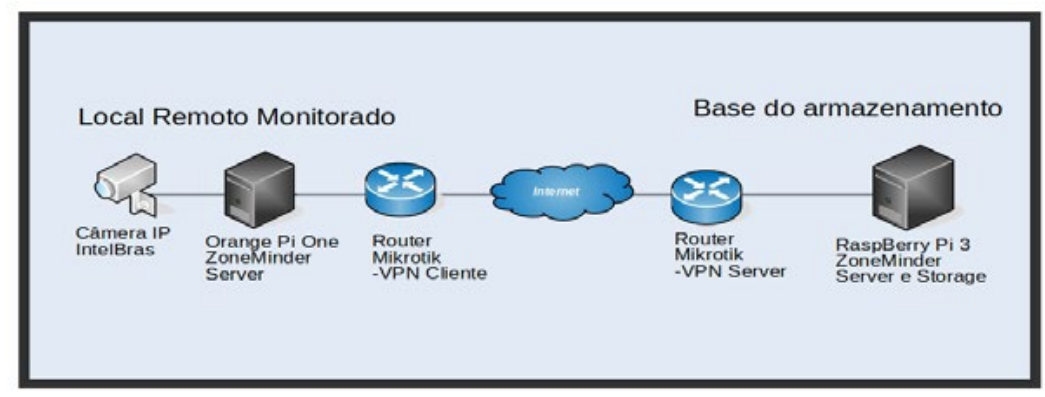

Fonte: Dos autores (2018). 
O protocolo de transmissão de imagem utilizado nesta câmera de segurança, o RTSP, torna o dispositivo um servidor de imagens. Dessa forma faz-se necessário que a porta de comunicação do serviço de streaming de vídeo da câmera esteja disponível para o servidor Zoneminder.

Somente a base de armazenamento remoto possui endereço de IP público, de modo que fosse possível a comunicação entre esses dispositivos, foi configurado um serviço de VPN entre os roteadores Mikrotik. Nessa VPN há uma terceira rede, de prefixo 172.17.10.0/24, ao adicionar as rotas para redes LAN remotas nos roteadores, permitiu que os dispositivos estabeleçam uma comunicação.

A Figura 5 demonstra o ambiente com a adição de um servidor Zoneminder operando como um analisador de imagem, com IP 192.168.85.2/24, instalado no local remoto monitorado. O restante do cenário permanece inalterado em relação ao descrito na Figura 4. Dessa forma a câmera IP instalada no local remoto monitorado envia as imagens em tempo real para este servidor instalado no mesmo local, que ao detectar movimento, envia as imagens e dados do evento para o servidor Zoneminder instalado na base de armazenamento.

Figura 5: Mapa de monitoramento no sistema Nagios

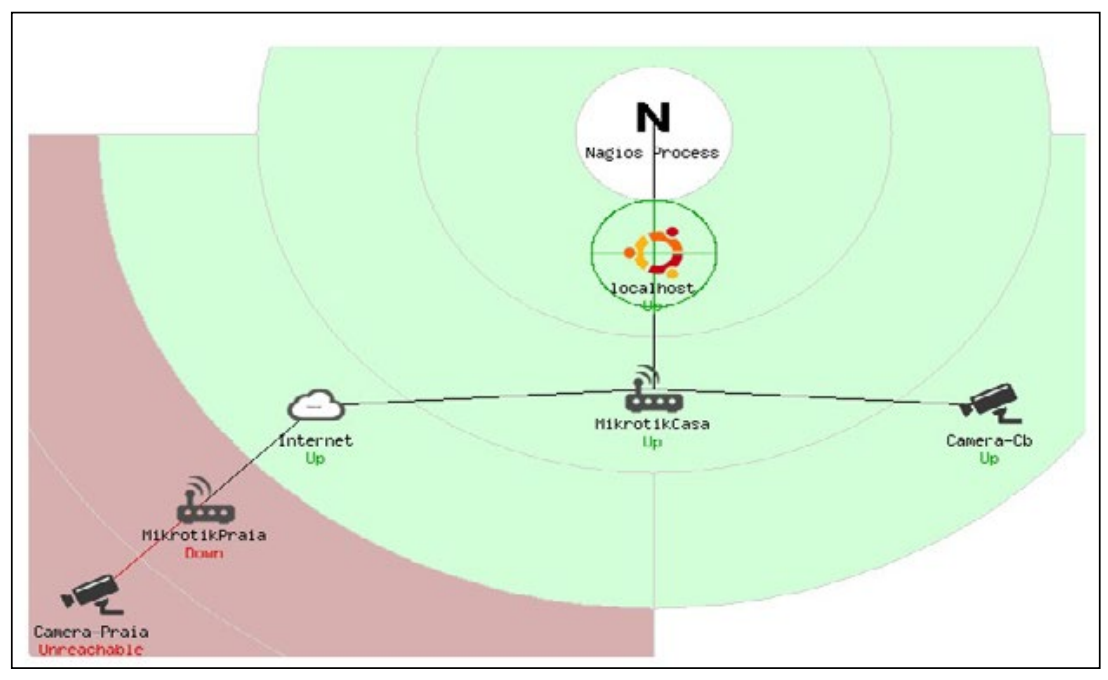

Fonte: Dos autores (2018).

Ainda que a otimização do fluxo de dados seja obtida, o sistema remoto requer uma conexão de dados ativa e permanente com o banco de dados do centralizador de imagens. Um dos requisitos para implantação deste sistema é o baixo custo, fazendo que a contratação de links redundantes não seja necessária. 
A falta de conexão torna o sistema inoperante no local remoto monitorado, para sanar este problema optou-se pela elaboração de uma rotina automatizada desenvolvida em Linux Bash para verificar o status da conexão com o banco de dados, tornando a aplicação de videomonitoramento remota um sistema independente na falta de comunicação com a base.

As imagens captadas nesse modo são armazenadas localmente, sendo transmitidas para uma conta de serviço de armazenamento em nuvem, ou quando a conexão de internet é restabelecida, conforme já mencionado anteriormente sobre a utilização do software Rclone. Assim que a conexão com banco de dados do centralizador de imagens é restabelecida, a mesma rotina automatizada desenvolvida de Linux Bash anteriormente citada verifica, fazendo com que a aplicação remota volte a processar somente a detecção de movimento, enviando as imagens novamente para o centralizador na base de armazenamento.

Da mesma forma que a utilização do software Rclone proporciona uma funcionalidade extra, a utilização do software Nagios para fins de monitoramento dos pontos remotos (Figura 5) permite a criação de gráficos de histórico de estado, notificações por e-mail de alterações de estados dos dispositivos, entre outros. Com o recebimento dessa notificação, possibilita com que o serviço seja restabelecido o mais rápido possível, pois o usuário não precisa acessar o sistema para constatar a existência de um problema, podendo, assim, providenciar a solução ao visualizar a notificação.

A aplicação Zoneminder também pode ser acessada através de um aplicativo gratuito para smartphones, porém com funcionalidades restritas e necessidade de IP público para acesso pela internet. Como a maioria das câmeras IP disponíveis no mercado possuem aplicativos específicos próprios para visualização de imagens através de internet, em nuvem, tornam-se uma opção mais prática. Essa funcionalidade das câmeras também permite fácil acesso às imagens, sem a necessidade de uma configuração complexa.

Outro aspecto de suma importância para elaboração deste sistema foi a plataforma de minicomputadores RaspberryPi, OrangePi ou outros modelos disponíveis no mercado de arquitetura semelhante, para instalação e configuração do sistema operacional, softwares como o Zoneminder, Nagios, Rclone. O fato de terem um bom custo-benefício, desempenho satisfatório e baixo consumo de energia contribuiu para esta definição. Essa última característica possibilita a utilização de um sistema de alimentação elétrica obtida através de energia solar de baixa potência e baterias pequenas. Juntamente com a utilização de redes Mesh, pode-se criar uma malha de rede grande, permitindo, por exemplo, monitorar uma propriedade rural extensa, dispensando a utilização de infraestrutura de cabeamento. 


\section{IMPLEMENTAÇÃO DA SOLUÇÃO}

Para elaboração das funcionalidades de transmissão das imagens para o serviço do Google Drive e a remoção das imagens após período estipulado foram utilizadas três rotinas implementadas em Linux Bash. A primeira rotina (Quadro 1) é responsável por iniciar a rotina de envio das imagens no boot do sistema operacional, garantindo a execução contínua da transmissão.

Quadro 1: Rotina de inicialização da rotina de envio

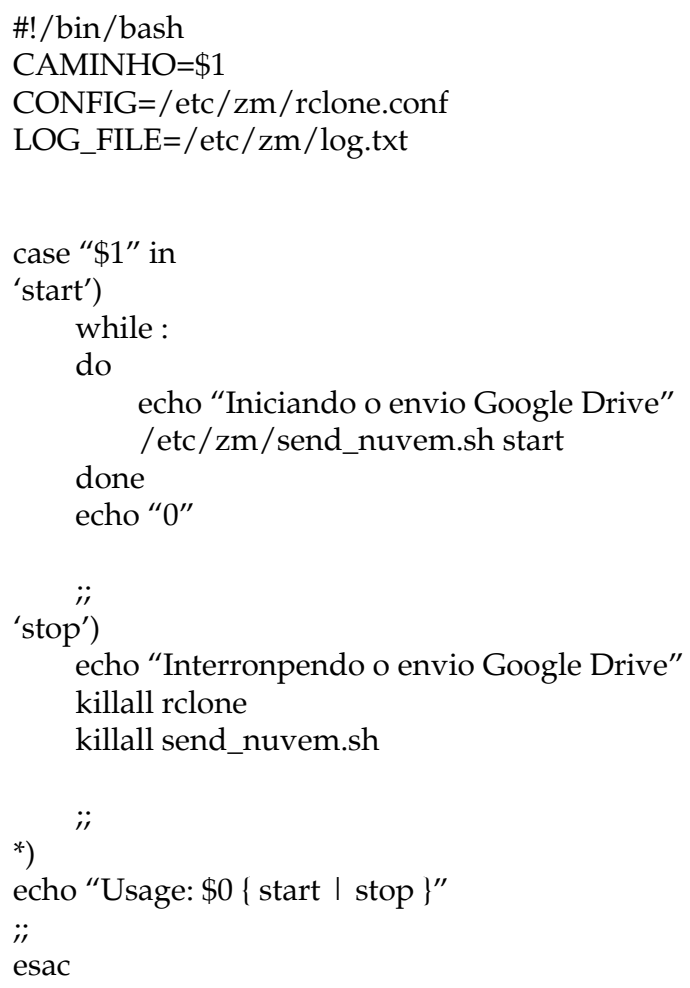

Fonte: Dos autores (2018).

A rotina para transmissão das imagens para o Google Drive, que executa apenas uma instância do aplicativo Rclone para evitar a sobrecarga do dispositivo, pode ser vista no Quadro 2. Sempre que a rotina é concluída, ela é executada sucessivamente para fins de garantir a transmissão dos dados o mais rápido possível. Esta rotina necessita ser executada em todos os servidores Zoneminder do sistema. 
Quadro 2: Rotina de envio das imagens para Google Drive

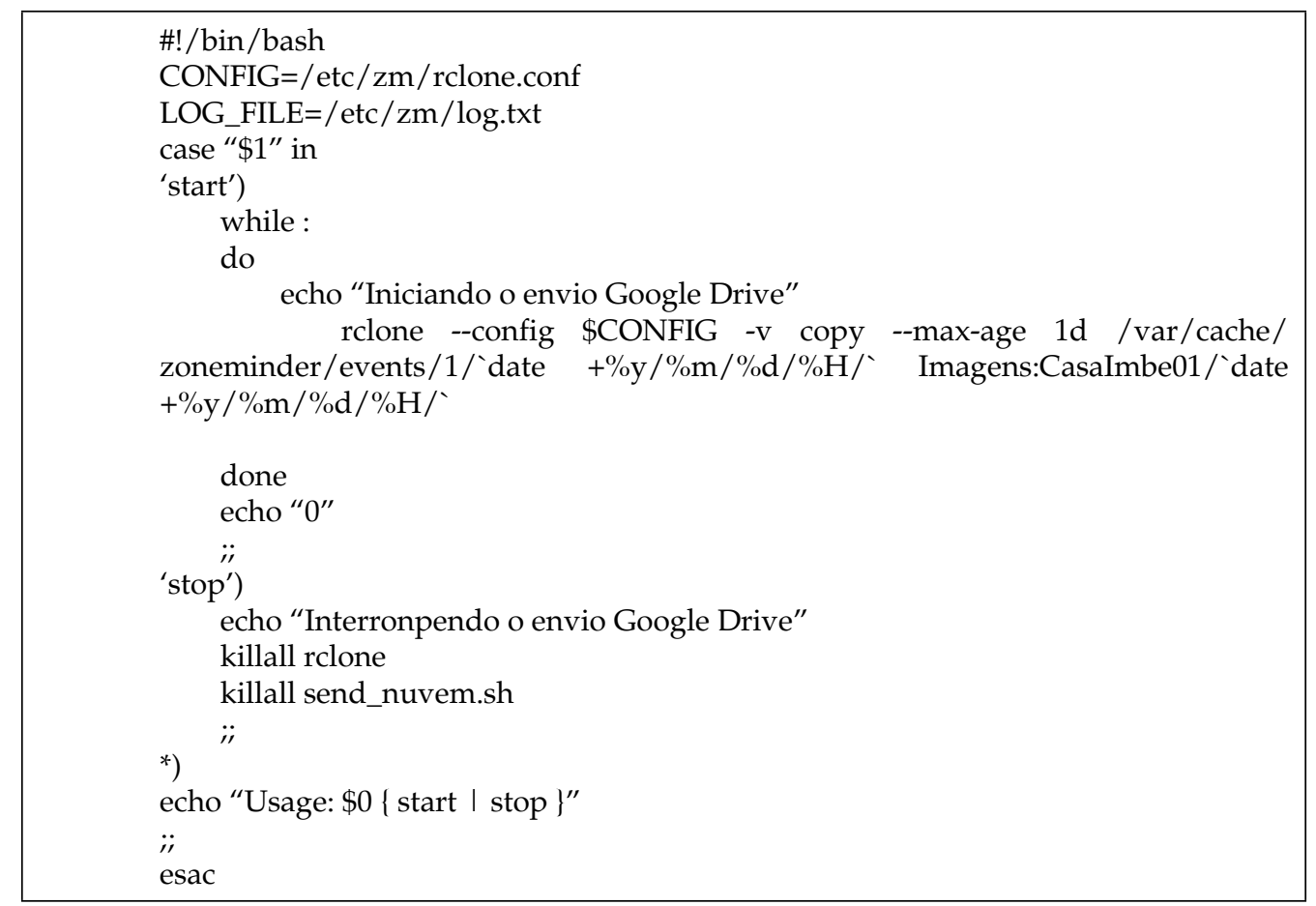

Fonte: Dos autores (2018).

A plataforma de serviço Google Drive oferece apenas 15 GBytes de armazenamento gratuito, para evitar que o sistema ocupe todo o recurso disponibilizado na conta do usuário, a rotina abaixo (Quadro 3) possibilita que imagens fiquem armazenadas conforme o período especificado pelo usuário. Esta rotina também é necessária que seja executada em todos os servidores Zoneminder do sistema, uma vez ao dia para evitar sobrecarga do minicomputador, adicionada na agendador de tarefas. 
Quadro 3: Rotina de exclusão das imagens antigas no Google Drive

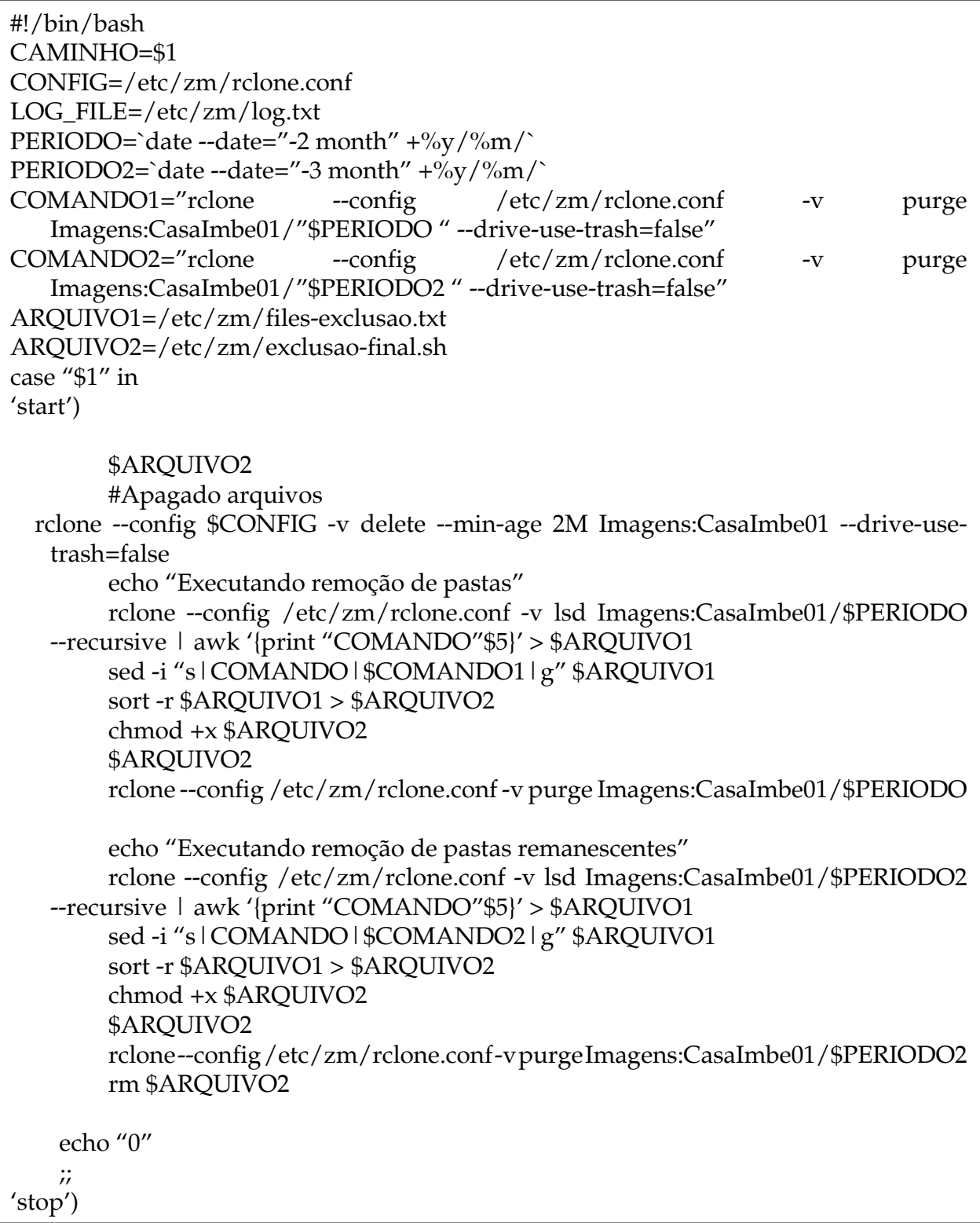

Fonte: Dos autores (2018).

Em caso de falta de conectividade do servidor de análise de detecção de movimento instalado no local remoto monitorado com o servidor da base de armazenamento, a rotina abaixo garante que as imagens sejam capturadas, armazenadas e transmitidas para a plataforma do Google Drive, assim que o servidor de análise de imagens tenha conexão com a internet. 
A rotina do Quadro 4 faz com que o servidor de análise de imagens instalado no local remoto monitorado possa trabalhar de forma independente na falta de conectividade e restabelecer o envio das informações para base de armazenamento assim que o problema de conectividade for sanado.

Quadro 4: Rotina de verificação de conectividade entre local remoto e base de armazenamento

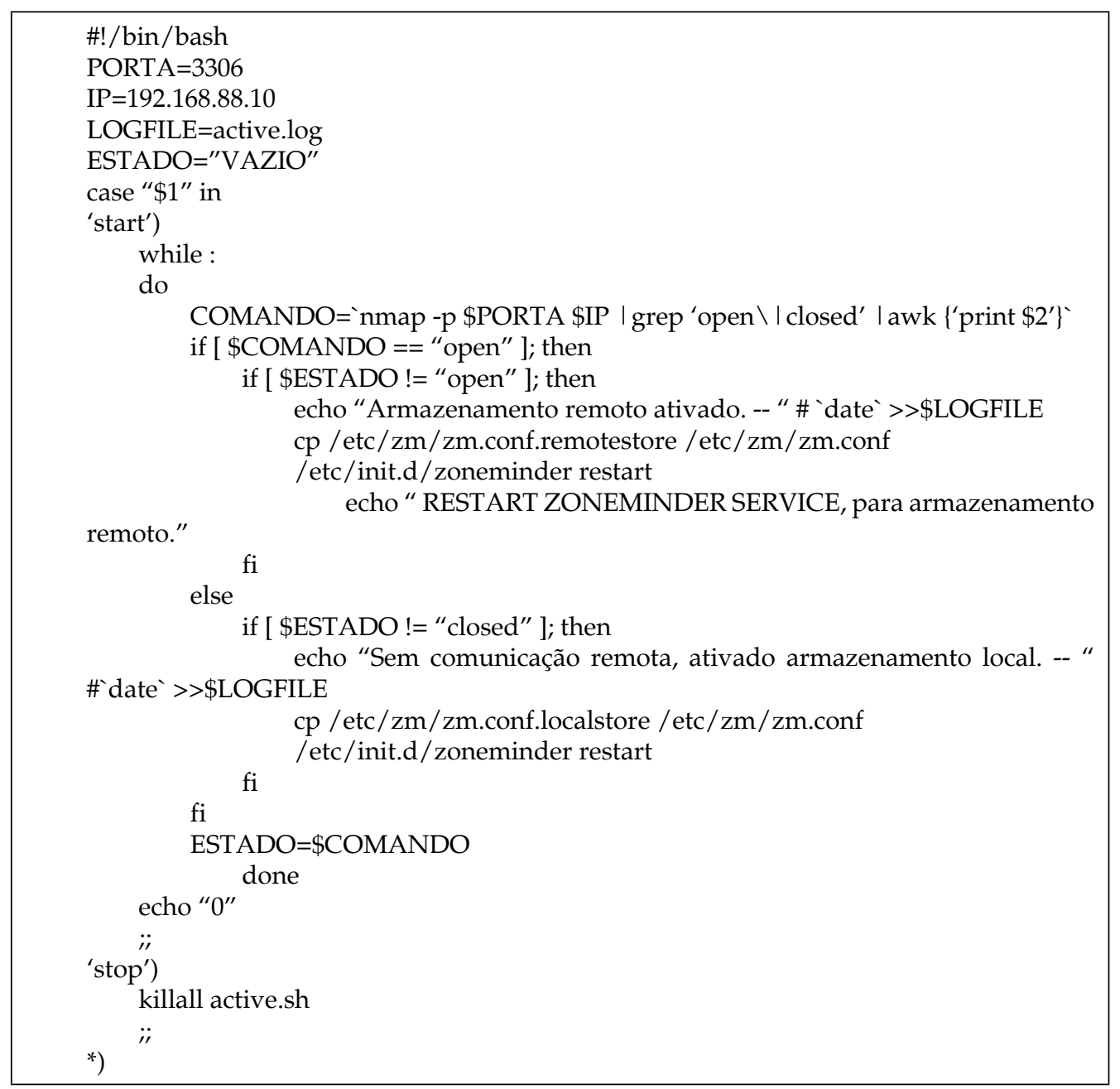

Fonte: Dos autores (2018).

A partir desta configuração o sistema ficou operante, garantido o armazenamento e/ou envio das imagens conforme as condições possíveis disponíveis de comunicação entre os dispositivos locais e remotos e internet. 


\section{RESULTADOS E DISCUSSÃO}

Nesta seção são demonstrados os resultados obtidos pela elaboração do referencial teórico e pela implementação do estudo de caso. Os testes foram realizados nos cenários 1 e 2, para ambos os cenários foram realizados medição de consumo de dados.

No primeiro cenário, conforme ilustrado na Figura 6, o servidor Zoneminder instalado na base de armazenamento remoto está configurado para capturar diretamente as imagens da câmera de segurança IP no local remoto monitorado. $\mathrm{O}$ teste deste cenário se consistiu em realizar a medição do tráfego da interface VPN do Mikrotik, onde constatou-se um consumo de aproximadamente 50 MBytes em apenas dez minutos de medição.

Figura 6: Início e final da medição do consumo de dados

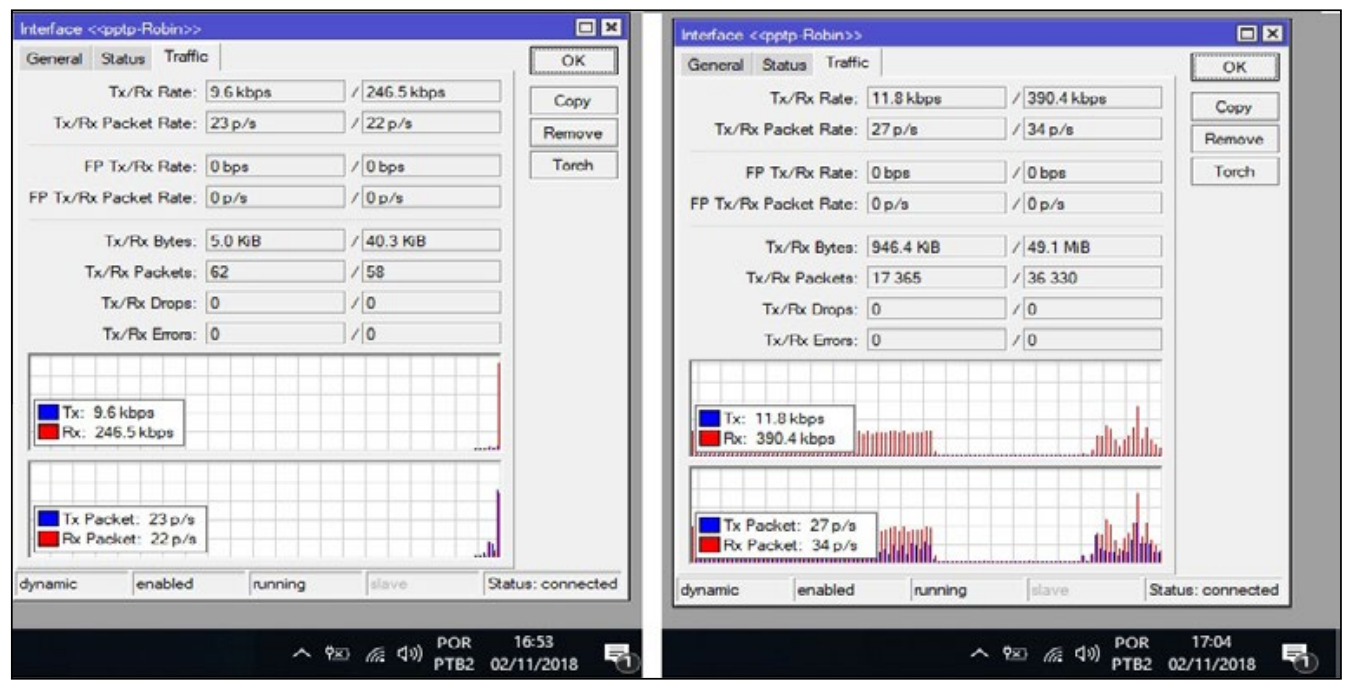

Fonte: Dos autores (2018).

A partir desses consumos foi calculado que em uma hora o sistema consumiria aproximadamente 300 MBytes, fazendo que o pacote de dados de 1 GBytes semanal contratado da operadora de telefonia móvel durasse apenas três horas e meia.

No segundo cenário, foi adicionado um segundo servidor Zoneminder, instalado no local remoto monitorado. Este novo servidor tem a função de capturar as imagens da câmera, analisá-las e enviá-las somente para o servidor Zoneminder instalado na base de armazenamento somente quando detectar movimentos.

$\mathrm{Na}$ medição do cenário anterior foi utilizado o Mikrotik para fazer a medição de consumo, porém nesse cenário o tempo de medição é maior e 
com a falta de energia o Mikrotik zera seus contadores. Para contornar esse problema, foi utilizado a medição de consumo de internet da operadora de telefonia móvel. Conforme indica a Figura 7, neste cenário o consumo de dados foi aproximadamente de apenas 290 MBytes em seis dias.

Figura 7: Medição do cenário 2, pelo sistema da operadora de telefonia móvel

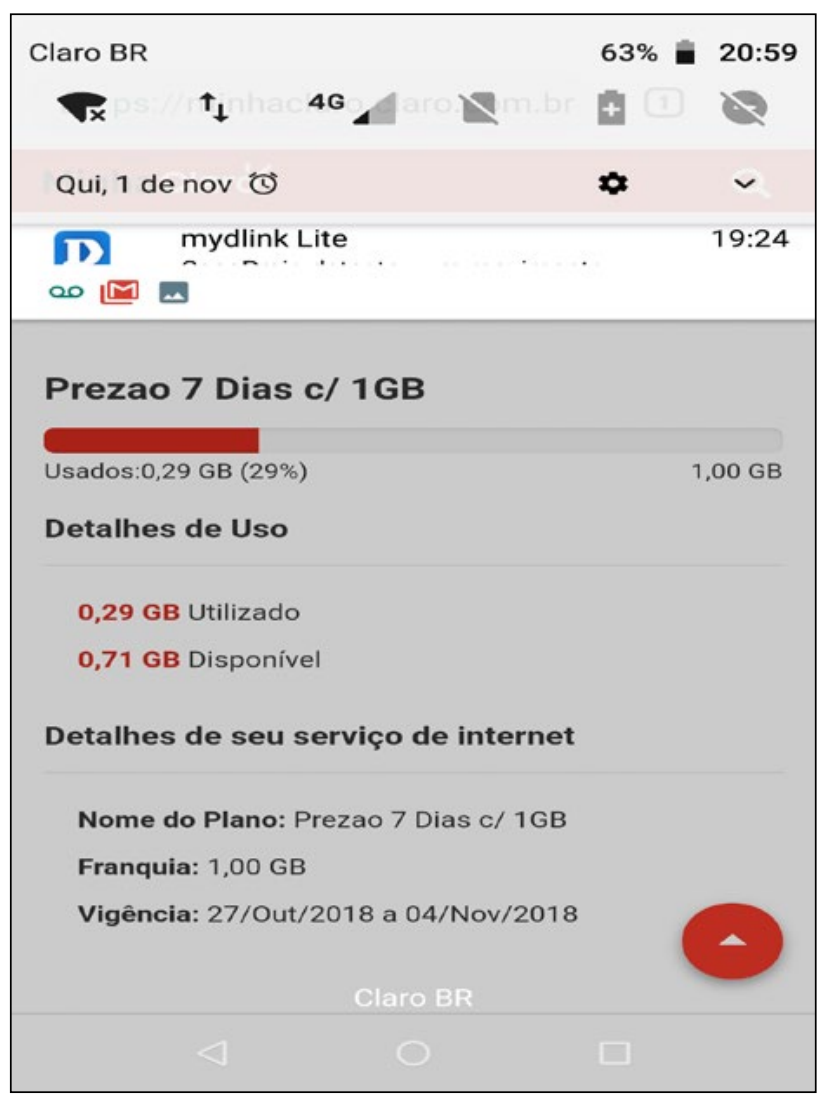

Fonte: Dos autores (2018).

O sistema se demonstrou estável e satisfatório, porém podemos apontar as seguintes limitações:

- A capacidade de processamento dos microcomputadores é limitada, fazendo com que a exibição de um evento registrado em uma resolução HD seja semelhante a um vídeo em câmera lenta.

- O filtro criado no Zoneminder, para remoção de imagens antigas ao atingir o percentual de utilização da capacidade de armazenamento de disco deve ser executado a cada uma hora, e o comando deve ser instalado na crontab do sistema operacional. 


\section{CONSIDERAÇÕES FINAIS}

O objetivo deste trabalho foi o de apresentar um sistema de videomonitoramento de baixo custo, com armazenamento remoto e centralizado sem a necessidade de contratação de uma estrutura para tal finalidade.

Apesar das limitações do hardware utilizado para o processamento das imagens, os objetivos foram alcançados, pois além do baixo custo para aquisição dos equipamentos, seu custo de manutenção do serviço também é baixo.

Além dessa vantagem, o sistema também se mostra escalável, permitindo desde a implantação de um pequeno sistema de videomonitoramento de pequeno porte para uma residência, até a construção de um sistema vigilância eletrônica de porte maior, com diversos servidores dedicados, como de banco de dados, armazenamento das imagens e processamento dos eventos.

Essa escalabilidade permite também a utilização otimizada de links de internet com franquia de dados limitada, enviando imagens para base de armazenamento somente quando necessário, contribuindo para que custo do sistema não fique elevado para o monitoramento de locais remotos.

Outra característica importante é a facilidade de adicionar novas funcionalidades, como é um computador rodando um sistema operacional Linux, que permite que funções deste sejam implementadas. Neste trabalho foi implementado o monitoramento dos ativos utilizando o software Nagios e o envio das imagens para armazenamento em nuvem para o Google Drive com o software Rclone, funcionalidade estas não presentes em diversos produtos disponíveis no mercado brasileiro.

\section{REFERÊNCIAS}

CLARO. Claro - Site operador de telefonia. Disponível em: <https:/ / www.claro.com. br/celular/planos-pre/prezao>. Acesso em: 10 set. 2018.

CORREA NETO, Alcides Dias. O Sistema de Videomonitorização como Ferramenta de Policiamento Preventivo. Disponível em: <http:/ / www.policiamilitar.sp.gov.br/ caes/artigos/Artigos\%20pdf/Alcides\%20Dias\%20Correa\%20Neto.pdf $>$. Acesso em: 1 nov. 2018.

FINATI, Rafael André - [Review] O Raspberry PI e os sistemas embarcados. Disponível em:<http:/ /www.actionnerds.com.br/review-o-raspberry-pi-e-ossistemas-embarcados/>. Acesso em: 26 set. 2018.

GIL, Antonio Carlos. Como elaborar projetos de pesquisa. 4. ed. São Paulo: Atlas, 2008.

GOGONI, Ronaldo - ASUS lança rival do Raspberry Pi que suporta 4K por US\$ 68.

Disponível em:<https://meiobit.com/359432/asus-tinker-board-mini-pc-concorrente- 
raspberry-pi-3-voltado-para-midia-com-suporte-a-4k-preco-um-tanto-salgado-68dolares/>. Acesso em: 10 set. 2018.

KUROSE, Jim; ROSS, Keith. Redes de computadores e a Internet - uma abordagem top-down. $6^{\text {a }}$ Ed. São Paulo: Pearson. 2015.

MORIMOTO, Carlos E. Raspberry Pi finalmente para pronta-entrega. Disponível em:<https:/ / www.hardware.com.br/noticias/2012-04/raspberry-ready.html $>$. Acesso em: 10 nov. 2018.

NAGIOS. Nagios - The Industry Standard In IT Infrastructure Monitoring. Disponível em: <https:/ / www.nagios.org/>. Acesso em: 10 nov. 2018.

Rclone. Rclone - Rsync for cloud storage. Disponível em: <https://rclone.org/>. Acesso em: 12 nov. 2018.

SIMÕES, Pedro - Já foram vendidos mais de $\mathbf{1 0}$ milhões Raspberry Pi. Disponível em:<https:/ / pplware.sapo.pt/gadgets/hardware/ja-vendidos-10-milhoes-raspberrypi/>. Acesso em: 09 set. 2018.

ZONEMINDER. Zoneminder - A full-featured, open source, state-of-the-art video surveillance software systemem. Disponível em: <https://zoneminder.com/>. Acesso em: 18 jul. 2018. 